\title{
Monitoring the operation of a graphene transistor in an integrated circuit by XPS
}

\author{
Pinar Aydogan a, Osman Balci ${ }^{\text {b }}$, Coskun Kocabas ${ }^{\text {b }}$, Sefik Suzer a, * \\ a Department of Chemistry, Bilkent University, 06800, Ankara, Turkey \\ ${ }^{\mathrm{b}}$ Department of Physics, Bilkent University, 06800, Ankara, Turkey
}

\section{A R T I C L E I N F O}

\section{Article history:}

Received 22 March 2016

Received in revised form

20 June 2016

Accepted 20 June 2016

Available online 2 July 2016

Keywords:

Graphene

XPS

Contact resistance

Integrated circuit

Transistor

\begin{abstract}
A B S T R A C T
One of the transistors in an integrated circuit fabricated with graphene as the current controlling element, is investigated during its operation, using a chemical tool, XPS. Shifts in the binding energy of $\mathrm{C} 1 \mathrm{~s}$ are used to map out electrical potential variations, and compute sheet resistance of the graphene layer, as well as the contact resistances between the metal electrodes. Measured shifts depend on lateral positions probed, as well as on polarity and magnitude of the gate-voltage. This non-contact and chemically specific characterization can be pivotal in diagnoses.
\end{abstract}

두 2016 Elsevier B.V. All rights reserved.

\section{Introduction}

After the discovery of the two-dimensional honeycomb structured carbon about a decade ago, colossal academic activity has been witnessed around a single or few-layers of graphene. [1,2] Even a greater activity has also been devoted to the technological developments in terms of incorporation of graphene to the existing and well-developed infra-structure. [3-6] For characterization of the materials involving graphene, and more importantly, the devices fabricated from them, in addition to electrical, chemical characterization is also needed. In that respect, most of the commonly utilized optical techniques can only give indirect information about the electrical properties seeked. [7-14] Similarly, information derived using the conventional and powerful electron spectroscopic techniques, like Auger (AES), Ultra-Violet (UPS), and $\mathrm{X}$-ray (XPS) photoelectron spectroscopies are also indirect, when it comes to electrical properties. [15-19] However, under appropriate conditions, all of the electron spectroscopic techniques are capable of reflecting local electrical potentials developed, intentionally or not, on the analyzed material or the device, since such potentials are embedded into the measured kinetic energy of the ejected

\footnotetext{
* Corresponding author

E-mail address: suzer@fen.bilkent.edu.tr (S. Suzer).
}

electrons. Accordingly, difference between the obtained binding energy and the tabulated one gives the chemically-addressed local electrical potential, which has been extensively utilized by our group and others to harvest electrical information, difficult to obtain by other methods. [20-23] Using this methodology, we have previously reported on analyses of a device using graphene as the resistive sheet between the two electrodes, [20] and in another device where the graphene layer was operated in the back-gated transistor geometry. [21] The present work extends it to analyze one of the transistors of the 64 elements of an IC-device, where C1s binding energy position is measured with lateral resolution to probe and map-out electrical properties of the transistor. Investigation of a single element out of the 64, is presented as "a proof of principle", since extension to the others are straightforward.

\section{Experimental details}

A picture of the device together with a survey XP spectrum of a graphene-only region is given in Fig. 1(a), where the dominating O1s feature belongs to the $\mathrm{SiO}_{2}$ substrate, since probe depth is about $10 \mathrm{~nm}$, but the $\mathrm{C} 1 \mathrm{~s}$ peak has the peak position of graphene at $284.7 \mathrm{eV}$. We synthesize single layer graphene on an ultra-smooth copper foil (purchased from Mitsui mining and smelting company, LTD, B1-SBS) by using a chemical vapor deposition (CVD) system connected to a vacuum pump. We heat up copper foils to $1035^{\circ} \mathrm{C}$ 


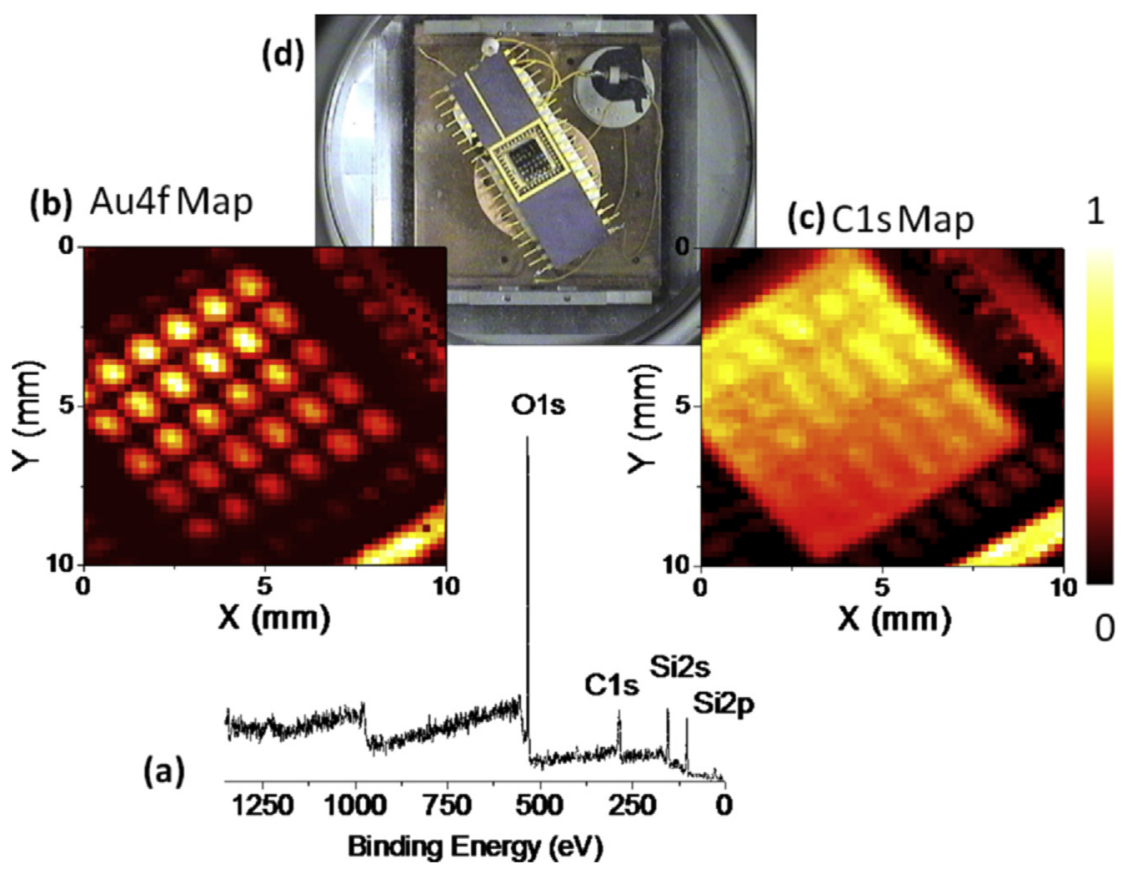

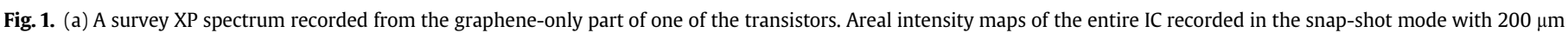
X-ray spot size and $200 \mu \mathrm{m}$ steps of; (b) Au4f, and (c) C1s. (d) Picture of the IC inserted into the load-lock of the instrument.

under $100 \mathrm{sccm} \mathrm{H}_{2}$ flow. We, then, grow graphene by flowing 10 sccm $\mathrm{CH}_{4}$ to the chamber for $1 \mathrm{~min}$ at that temperature. The partial pressures of the $\mathrm{H}_{2}$ and $\mathrm{CH}_{4}$ are 1.5 Torr and 3 Torr, respectively, during the growth process. We cool down the chamber at a rate of $10^{\circ} \mathrm{C} / \mathrm{s}$ to room temperature, while $100 \mathrm{sccm} \mathrm{H}_{2}$ flows. To transfer graphene on $\mathrm{SiO}_{2}$ substrates, we first coat the copper foil with the Shipley 1806 resist and dry them by annealing overnight at $80{ }^{\circ} \mathrm{C}$. Then, we etch the graphene grown copper in $\mathrm{FeCl}_{3}$ aqueous solution. After washing and drying the resist-graphene stack, we put it on $\mathrm{SiO}_{2}$ substrate and heat the resist-graphene- $\mathrm{SiO}_{2}$ stack at $70{ }^{\circ} \mathrm{C}$ for $2 \mathrm{~min}$ and at $120^{\circ} \mathrm{C}$ for $20 \mathrm{~s}$. This process ensures that the resistgraphene stack perfectly sticks to the $\mathrm{SiO}_{2}$ surface. We dissolve the dry resist with acetone and finally obtain a graphene transferred on $\mathrm{SiO}_{2}$ substrate. To fabricate back-gated graphene transistors on $\mathrm{Si}^{+} /$ $\mathrm{SiO}_{2}$ substrate, we evaporate $50 \mathrm{~nm}$ Au for source and drain contacts to the graphene. After isolating each transistor channel with a reactive ion etching (RIE) process, we evaporate $5 \mathrm{~nm}-\mathrm{Ti}$ and
$100 \mathrm{~nm}-\mathrm{Au}$ to extend the area of source and drain contacts.

Mechanically strong metal contacts are required for wirebonding. The fabricated transistors used in this work have $0.5 \mathrm{~mm}$ channel width and $1.0 \mathrm{~mm}$ channel length. Schematic drawing of the fabricated transistors is shown in Fig. 2(a). To measure the electrical performance of the fabricated transistors, we use HP-4153 probe station.

\section{Results and discussion}

We apply $-1 \mathrm{~V}$ bias between the source and drain electrodes and measure the current as a function of the gate voltage, shown in Fig. 2(b). The result of the electrical-only measurements indicates the Dirac point to be near $0 \mathrm{~V}$, and other Current-Voltage characteristics are depicted in Fig. 2(c). The calculated field effect mobility of the device used in this study is around 820 and $600 \mathrm{~cm}^{2} / \mathrm{V}$ for holes and electrons, respectively (see the Supplementary
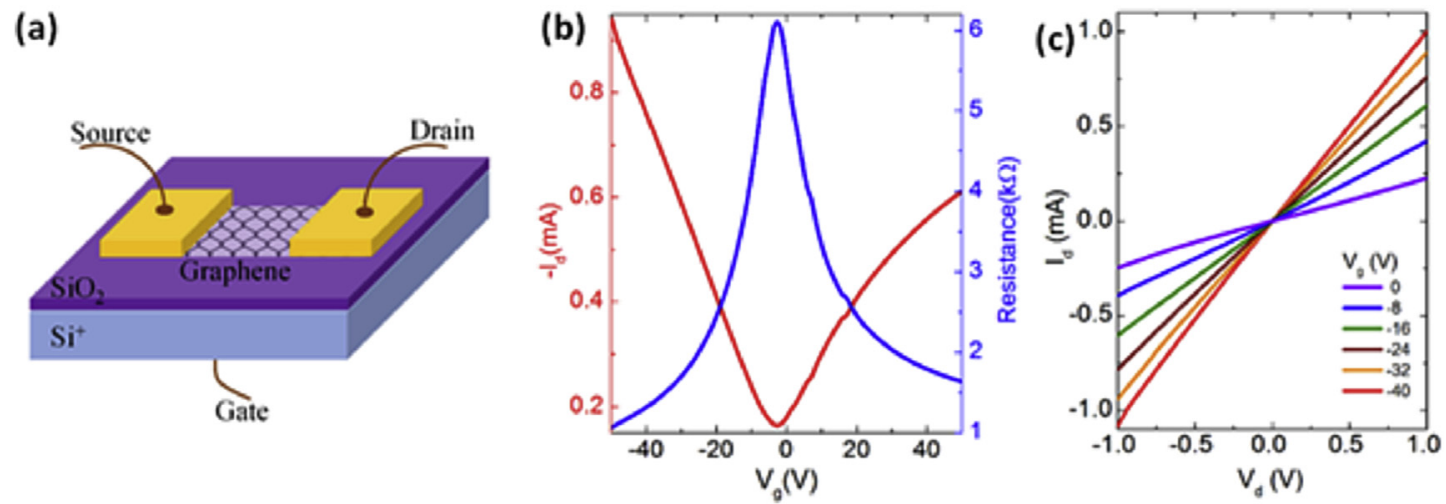

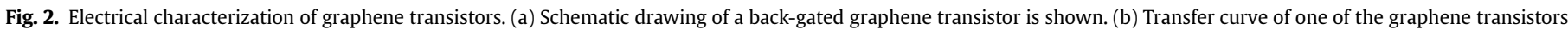

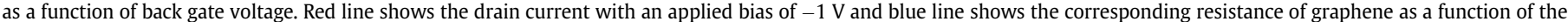

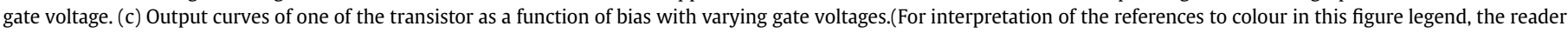
is referred to the web version of this article.) 
(a)

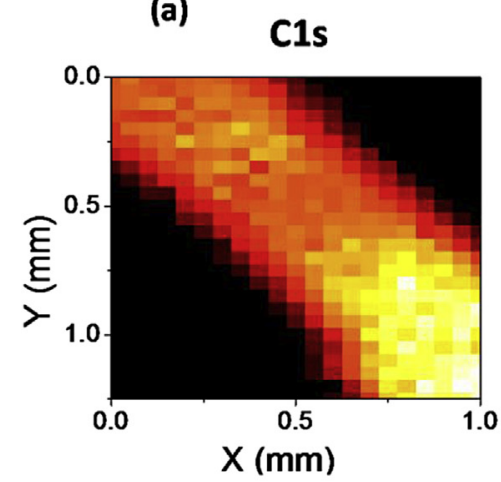

(d)

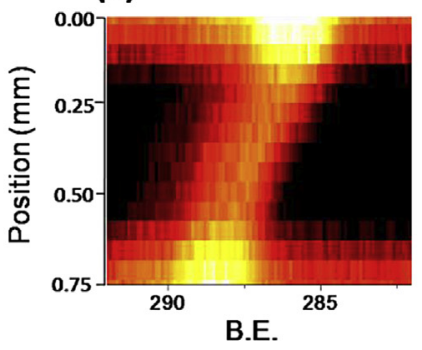

Vs

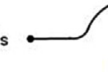

(b)

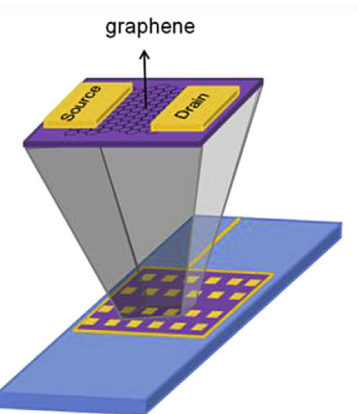

(e)

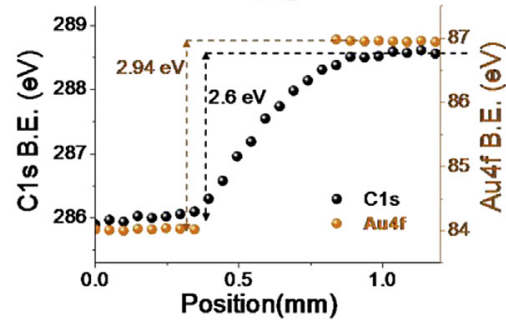

(c)

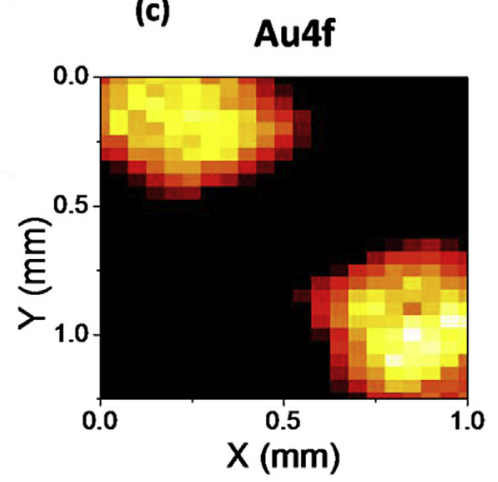

(f)

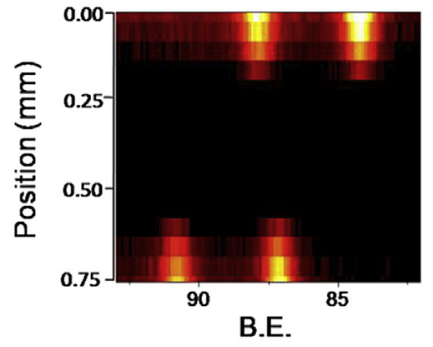

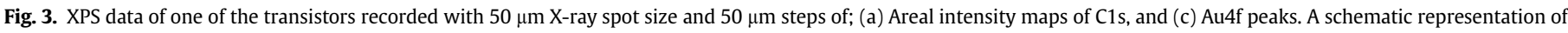

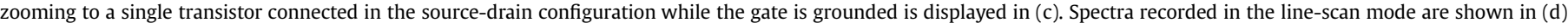
and (f), while the binding energy positions of the $\mathrm{C} 1 \mathrm{~s}$ and $\mathrm{Au} 4 \mathrm{f}_{7 / 2}$ are displayed in (e).

Information (SI) section and Fig. S1), where the mobility is calculated using the maximum transconductance of the device. Once the integrity of the transistors has been established by electrical measurements, further contacts are attached for XPS characterization, a picture of which was given in Fig. 1. The entire IC is inserted into the analysis chamber of the Thermo Fisher K-Alpha X-ray photoelectron spectrometer, which uses monochromatic AlK $\alpha$ ( $\mathrm{h} \nu=1486.6 \mathrm{eV}$ ) as the excitation source. Some of the measurements are done only in the source-drain, while the other in the source-drain-gate geometry. Therefore, the instrument is modified to provide two external voltage biases, where one is used as the source and the other as the gate, while the drain is grounded. Using the intensity of the $01 \mathrm{~s}$ peak of the substrate and the C1s of graphene from the survey spectrum of the graphene-only region shown in Fig. 1, it is possible to estimate the thickness of the graphene layer as $\sim 1.1 \mathrm{~nm}$. Computational details are given in the SI section.

In Fig. 1, areal intensity (computed area) maps of the entire IC recorded in the snap-shot mode with $200 \mu \mathrm{m}$ X-ray spot size and $200 \mu \mathrm{m}$ steps are also shown for the Au $4 \mathrm{f}$ and C1s peaks. Since the probe depth of XPS is about $10 \mathrm{~nm}$, gold electrodes and also the gold wire connections are easily identified, while carbon is everywhere. It is also possible to record the data with smaller spot size (50 $\mu \mathrm{m})$ of and steps $(50 \mu \mathrm{m})$, and zooming to only one of the transistors, as shown in Fig. 3, where the intensity of the C1s and Au4f are shown as areal maps (a) and (c). In the same Figure the peak positions are shown as regular scanned spectra recorded in the line-scan mode with inherently better precision in (d) and (f), while $\mathrm{a}+3 \mathrm{~V}$ bias is applied to the source electrode, and both the drain and the gate are grounded.

The extracted binding energies are shown in Fig. 3(e) for both peaks as a function of lateral position, and the actual spectra are reproduced in the SI section as Fig. S3. The binding energy of the $\mathrm{Au}_{4 \mathrm{f}} \mathrm{f}_{2}$ peak, which is commonly used as a reference, is $84.00 \mathrm{eV}$, and it is exactly what is measured at the grounded electrode. However, it is measured as $86.94 \mathrm{eV}$ at the source, yielding a difference of $2.94 \mathrm{eV}$ which reflects faithfully the applied voltage bias of $+3 \mathrm{~V}$, within the precision of our measurements. The measured difference in the position of the $\mathrm{C} 1 \mathrm{~s}$ peak across the electrodes is less precise and is $2.6 \mathrm{eV}$, i.e. $0.3 \mathrm{eV}$ less. This small but significant difference can be attributed to the contact resistance(s) between the graphene and the gold electrodes, since application of $3 \mathrm{~V}$ causes a sizable current of $\sim 2.6 \mathrm{~mA}$, passing between the metal electrodes, consistent with the geometry of the graphene layer. The contact resistance, especially in devices with smaller dimensions is known to adversely affect the performance. [24-30] Therefore, location, as well as information related with its chemical nature is highly desirable. Besides the conventional electrical-only measurements, Kelvin probe force microscopy is the only other tool which can locate and quantify contact resistance(s), [27] but again it, too, falls short when it comes to chemical specificity. Hence use of XPS for this purpose is very unique.

In order to harvest the effect of gating, instead of applying a voltage bias, a current bias needs to be imposed, since the gate is the current controlling element of the circuit, which requires two biases. That is what is implemented for the data shown in Fig. 4, where a constant voltage bias of $+3 \mathrm{~V}$ is applied to the source through a precision $1 \mathrm{k} \Omega$ series resistor, and a second variable voltage bias is applied to the gate, while grounding the drain, as schematically shown in the same figure. Here again, the voltage drop across the gold electrodes, as well as the potential variations through the graphene sheet, under three different gate voltages, are measured through the positions of the $A u 4 f_{7 / 2}$ and $\mathrm{C} 1 \mathrm{~s}$ peaks, respectively, which are given in Table 1 . Using these data together with a simple equivalent circuit model, both the sheet as well as the contact resistances can be obtained for all 4 different operational conditions of the transistor, in an all non-invasive fashion (details are given in the SI section). The values obtained are consistent with 
(a)

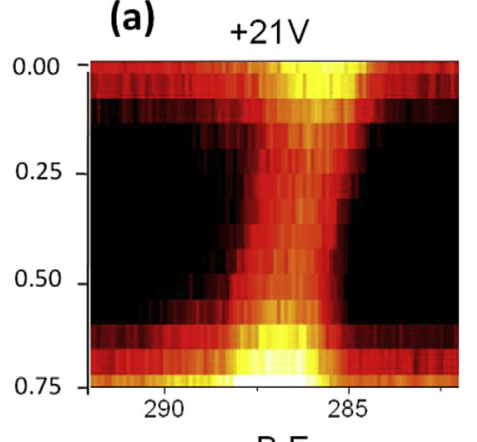

B.E. (b)

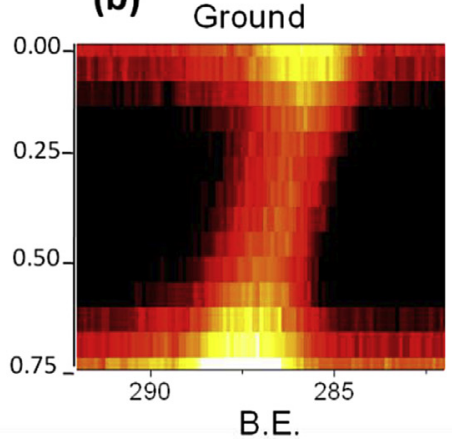

(c)

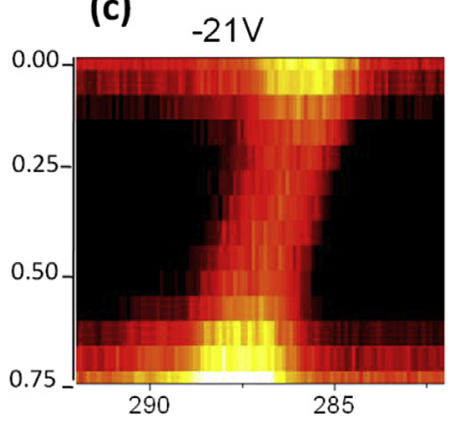

B.E.

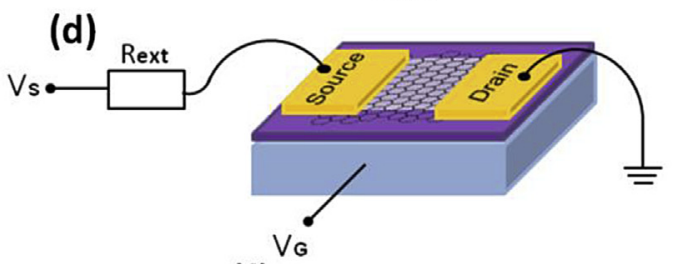

(e)

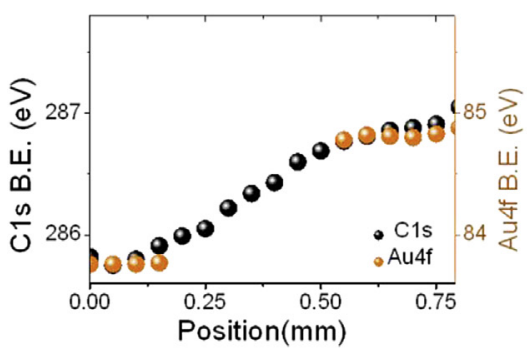

(f)

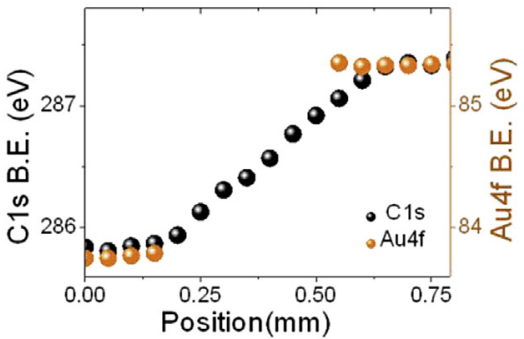

(g)

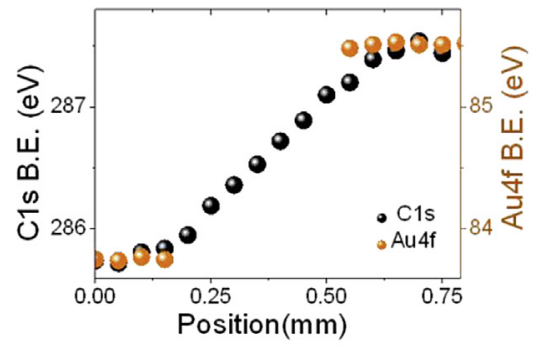

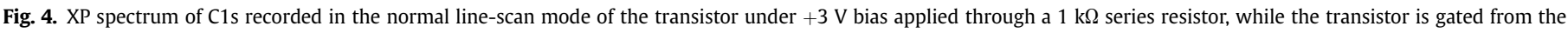

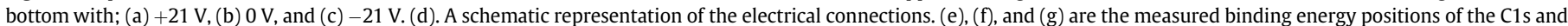
Au4f $f_{7 / 2}$ for the corresponding data in (a), (b), and (c).

Table 1

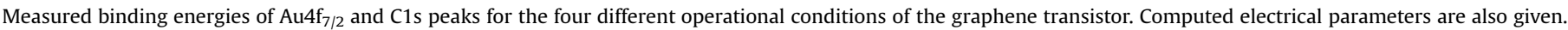
Details of which are given in the SI section.

\begin{tabular}{|c|c|c|c|c|c|c|c|c|c|c|}
\hline & \multirow[t]{2}{*}{ Gate (V) } & \multicolumn{3}{|c|}{ B.E. $\left(A u 4 f_{7 / 2}\right)(e V)$} & \multicolumn{3}{|c|}{ B. E (C1s) $(\mathrm{eV})$} & \multirow[t]{2}{*}{ Sheet resistance $(\Omega)$} & \multirow[t]{2}{*}{ IR drop (eV) } & \multirow[t]{2}{*}{ Contact resistance $(\Omega)$} \\
\hline & & $\mathrm{S}$ & $\mathrm{D}$ & $\Delta$ & $\mathrm{S}$ & $\mathrm{D}$ & $\Delta$ & & & \\
\hline No gating (+3 V Bias) & - & 86.94 & 84.00 & 2.94 & 287.5 & 284.9 & 2.6 & $1020(1.2 \mathrm{k} \Omega / \mathrm{sq})$ & 0.34 & $130(52 \mathrm{k} \Omega \mu \mathrm{m})$ \\
\hline \multirow[t]{3}{*}{$+3 \mathrm{~V}$ over $1 \mathrm{k} \Omega$ Series Resistor } & 0 & 85.57 & 84.00 & 1.57 & 286.2 & 284.8 & 1.4 & $1020(1.2 \mathrm{k} \Omega / \mathrm{sq})$ & 0.17 & $120(48 \mathrm{k} \Omega \mu \mathrm{m})$ \\
\hline & +21 & 86.12 & 84.00 & 1.12 & 285.8 & 284.8 & 1.0 & $550(0.6 \mathrm{k} \Omega / \mathrm{sq})$ & 0.12 & $65(26 \mathrm{k} \Omega \mu \mathrm{m})$ \\
\hline & -21 & 86.75 & 84.00 & 1.82 & 286.5 & 284.8 & 1.7 & $1550(1.6 \mathrm{k} \Omega / \mathrm{sq})$ & 0.12 & $65(26 \mathrm{k} \Omega \mu \mathrm{m})$ \\
\hline
\end{tabular}

our previous electrical-only measurements, [29] and also with reported ones. It must also be stressed that extracted values of the transistor using XPS, as given in Table 1, differs from those depicted in Fig. 2 using electrical means. Such a difference is common since, the XPS measurements are performed in vacuum, in the absence of air and humidity, whereas the electrical measurements are carried out in air ambient, and the position of the Dirac point can deviate significantly between the two diverse conditions.

\section{Conclusions}

In summary, through application of electrical bias, XPS is shown to be able to extract electrical parameters of an isolated graphenebased transistor of a 64-element IC during its operation in a chemically specific and non-contact fashion.

\section{Acknowledgments}

This work is supported by the 2Dfun Project in the FLAG-ERA Joint Translational Call, 2015 within the Graphene Flagship and also by TUBITAK, the Scientific and Technological Research Council of Turkey, through the Grant No. $215 Z 534$.

\section{Appendix A. Supplementary data}

Supplementary data related to this article can be found at http:// dx.doi.org/10.1016/j.orgel.2016.06.027

\section{References}

[1] K.S. Novoselov, A.K. Geim, S.V. Morozov, D. Jiang, M.I. Katsnelson, I.V. Grigorieva, et al., Two-dimensional gas of massless Dirac fermions in graphene, Nature 438 (2005) 197-200.

[2] A.K. Geim, K.S. Novoselov, The rise of graphene, Nat. Mater. 6 (2007) 183-191. 
[3] S. Bae, H. Kim, Y. Lee, X. Xu, J.S. Park, Y. Zheng, et al., Roll-to-roll production of 30-inch graphene films for transparent electrodes, Nat. Nanotechnol. 5 (2010) $574-578$.

[4] F. Wang, Y. Zhang, C. Tian, C. Girit, A. Zettl, M. Crommie, et al., Gate-variable optical transitions in graphene, Science 320 (2008) 206-209.

[5] C. Berger, Z. Song, T. Li, X. Li, A.Y. Ogbazghi, R. Feng, et al., Ultrathin epitaxial graphite: 2d electron gas properties and a route toward graphene-based nanoelectronics, J. Phys. Chem. B 108 (2004) 19912-19916.

[6] Y.-M. Lin, A. Valdes-Garcia, S.-J. Han, D.B. Farmer, I. Meric, Y. Sun, et al., Waferscale graphene integrated circuit, Science 332 (2011) 1294-1297.

[7] A.C. Ferrari, J.C. Meyer, V. Scardaci, C. Casiraghi, M. Lazzeri, F. Mauri, et al., Raman spectrum of graphene and graphene layers, Phys. Rev. Lett. 97 (2006) 187401.

[8] Z.Q. Li, E.A. Henriksen, Z. Jiang, Z. Hao, M.C. Martin, P. Kim, et al., Dirac charge dynamics in graphene by infrared spectroscopy, Nat. Phys. 4 (2008) 532-535.

[9] M.M. Lucchese, F. Stavale, E.H.M. Ferreira, C. Vilani, M.V.O. Moutinho, R.B. Capaz, et al., Quantifying ion-induced defects and Raman relaxation length in graphene, Carbon 48 (2010) 1592-1597.

[10] L.G. Cancado, A. Jorio, E.H.M. Ferreira, F. Stavale, C.A. Achete, R.B. Capaz, et al, Quantifying defects in graphene via Raman spectroscopy at different excitation energies, Nano Lett. 11 (2011) 3190-3196.

[11] F.H.L. Koppens, D.E. Chang, F.J. Garcia de Abajo, Graphene plasmonics: a platform for strong light-matter interactions, Nano Lett. 11 (2011) 3370-3377.

[12] A. Eckmann, A. Felten, A. Mishchenko, L. Britnell, R. Krupke, K.S. Novoselov, et al., Probing the nature of defects in graphene by Raman spectroscopy, Nano Lett. 12 (2012) 3925-3930.

[13] M. Liu, X. Yin, X. Zhang, Double-layer graphene optical modulator, Nano Lett. 12 (2012) 1482-1485.

[14] E.O. Polat, C. Kocabas, Broadband optical modulators based on graphene supercapacitors, Nano Lett. 13 (2013) 5851-5857.

[15] T. Ohta, A. Bostwick, J.L. McChesney, T. Seyller, K. Horn, E. Rotenberg, Interlayer interaction and electronic screening in multilayer graphene investigated with angle-resolved photoemission spectroscopy, Phys. Rev. Lett. 98 (2007) 206802.

[16] A. Bostwick, T. Ohta, T. Seyller, K. Horn, E. Rotenberg, Quasiparticle dynamics in graphene, Nat. Phys. 3 (2007) 36-40.
[17] J. Wintterlin, M.L. Bocquet, Graphene on metal surfaces, Surf. Sci. 603 (2009) $1841-1852$

[18] T. Filleter, K.V. Emtsev, T. Seyller, R. Bennewitz, Local work function measurements of epitaxial graphene, Appl. Phys. Lett. 93 (2008) 133117.

[19] L.I. Johansson, S. Watcharinyanon, A.A. Zakharov, T. Iakimov, R. Yakimova, C. Virojanadara, Stacking of adjacent graphene layers grown on C-face SiC, Phys. Rev. B 84 (2011) 125405.

[20] C. Kocabas, S. Suzer, Probing voltage drop variations in graphene with photoelectron spectroscopy, Anal. Chem. 85 (2013) 4172-4177.

[21] M. Copuroglu, P. Aydogan, E.O. Polat, C. Kocabas, S. Suzer, Gate-tunable photoemission from graphene transistors, Nano Lett. 14 (2014) 2837-2842.

[22] B. Bozzini, M. Amati, L. Gregoratti, M.K. Abyaneh, M. Prasciolu, A.L. Trygub, et al., Microscale evolution of surface chemistry and morphology of the key components in operating hydrocarbon-fuelled SOFCs, J. Phy. Chem. C 116 (2012) 23188-23193.

[23] E.J. Crumlin, H. Bluhm, Z. Liu, In situ investigation of electrochemical device using ambient pressure photoelectron spectroscopy, J. Electron. Spectrosc. Relat. Phenom. 190 Part A (2013) 84-92.

[24] A. Javey, R. Tu, D.B. Farmer, J. Guo, R.G. Gordon, H. Dai, High Performance ntype carbon nanotube field-effect transistors with chemically doped contacts, Nano Lett. 5 (2005) 345-348.

[25] J. Cayssol, B. Huard, D. Goldhaber-Gordon, Contact resistance and shot noise in graphene transistors, Phys. Rev. B 79 (2009) 075428.

[26] A. Venugopal, L. Colombo, E.M. Vogel, Contact resistance in few and multilayer graphene devices, Appl. Phys. Lett. 96 (2010) 013512.

[27] J.A. Robinson, M. LaBella, M. Zhu, M. Hollander, R. Kasarda, Z. Hughes, K. Trumbull, R. Cavalero, D. Snyder, Contacting graphene, Appl. Phys. Lett. 98 (2011) 053103.

[28] L. Yan, C. Punckt, I.A. Aksay, W. Mertin, G. Bacher, Local voltage drop in a single functionalized graphene sheet characterized by Kelvin probe force microscopy, Nano Lett. 11 (2011) 3543-3549.

[29] O. Balci, C. Kocabas, Rapid thermal annealing of graphene-metal contact, Appl Phys. Lett. 101 (2012) 243105.

[30] P. Aydogan, E. Arslan, S. Cakmakyapan, E. Ozbay, W. Strupinski, S. Suzer, Voltage contrast X-ray photoelectron spectroscopy reveals graphenesubstrate interaction in graphene devices fabricated on the C- and Si-faces of SiC, Appl. Phys. Lett. 107 (2015) 121603. 